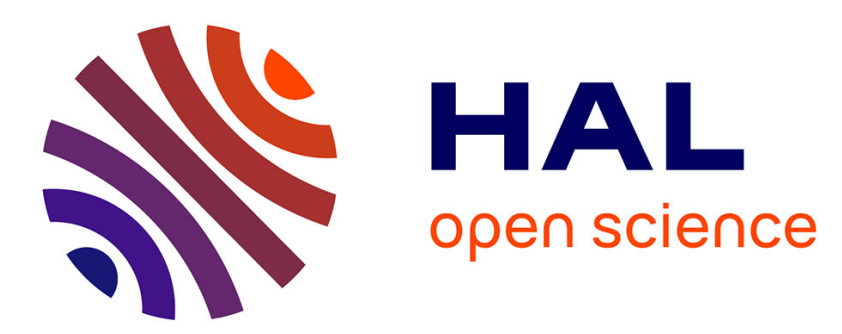

\title{
New data model for graph-cut segmentation: application to automatic melanoma delineation
}

Razmig Kéchichian, Hao Gong, Marinette Revenu, Olivier Lézoray, Michel

Desvignes

\section{> To cite this version:}

Razmig Kéchichian, Hao Gong, Marinette Revenu, Olivier Lézoray, Michel Desvignes. New data model for graph-cut segmentation: application to automatic melanoma delineation. ICIP 2014 - 21st IEEE International Conference on Image Processing, Oct 2014, Paris, France. hal-01080049

\section{HAL Id: hal-01080049 \\ https://hal.science/hal-01080049}

Submitted on 4 Nov 2014

HAL is a multi-disciplinary open access archive for the deposit and dissemination of scientific research documents, whether they are published or not. The documents may come from teaching and research institutions in France or abroad, or from public or private research centers.
L'archive ouverte pluridisciplinaire HAL, est destinée au dépôt et à la diffusion de documents scientifiques de niveau recherche, publiés ou non, émanant des établissements d'enseignement et de recherche français ou étrangers, des laboratoires publics ou privés. 


\title{
NEW DATA MODEL FOR GRAPH-CUT SEGMENTATION: APPLICATION TO AUTOMATIC MELANOMA DELINEATION
}

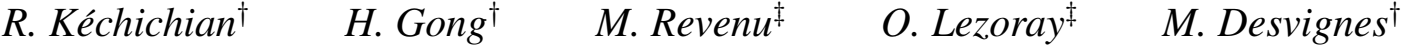 \\ † Gipsa-lab: CNRS UMR 5216; Université de Grenoble, Saint-Martin-d'Hères, France \\ $\ddagger$ Greyc-Image: CNRS UMR 6072; Ensicaen, Université de Caen, Caen, France
}

\begin{abstract}
We propose a new data model for graph-cut image segmentation, defined according to probabilities learned by a classification process. Unlike traditional graph-cut methods, the data model takes into account not only color but also texture and shape information. For melanoma images, we also introduce skin chromophore features and automatically derive "seed" pixels used to train the classifier from a coarse initial segmentation. On natural images, our method successfully segments objects having similar color but different texture. Its application to melanoma delineation compares favorably to manual delineation and related graph-cut segmentation methods.
\end{abstract}

Index Terms - image segmentation, melanoma, texture, shape, graph cut

\section{INTRODUCTION}

Melanoma is a malignant tumor of melanocytes. Although less common than basal and squamous cell cancers, it is the most fatal skin cancer which causes a considerable number of deaths [1]. Since treatment for metastatic melanoma is difficult, early diagnosis and prompt excision are critical to increase patient survival rate. Several diagnosis systems for melanoma have been defined, such as the ABCDE system [2] which uses a checklist of five parameters: asymmetry, border, color, dimension and evolution, and the 7-points checklist [3] which is a scoring approach of different features comprising color, shape and texture. Improvements in diagnostic sensitivity and specificity have been reported with the introduction of dermoscopy [4]. However, the interpretation of dermoscopic images is time-consuming, subjective, and lacks accuracy and reproducibility. Therefore, there is great interest in developing computer-aided diagnosis systems for melanoma.

Automatic dermoscopic image analysis typically includes four stages: 1) image acquisition, 2) image segmentation, 3) feature extraction and selection and 4) lesion classification. The segmentation stage, which delineates the borders of pigmented skin lesions (PSL), is of great importance since several important features, such as lesion dimension, shape, border irregularity, asymmetry etc. which provide important information for accurate diagnosis, are calculated on the delin- eated lesion border [5]. However, automatic accurate PSL delineation is a challenging task due low contrast between the lesion and surrounding skin, variegated pigmentation inside the lesion, fuzzy and irregular lesion boundaries and the presence of artifacts like hair, skin flakes and specular reflection.

Several methods have been developed for automatic or semi-automatic PSL delineation on melanoma images which mostly rely on color as being the most important information. Many systems select a simple scalar feature such as the intensity [6], or the $B$ and $b$ channel values of $R G B$ and $C I E$ $L * a * b *$ color spaces [7] which better discriminate in most dermoscopic images. Others use the CIEL $u^{*} u^{*}$ color model as a feature space [8], or its principal components [9] and luminance [10] information.

Classical segmentation techniques have been applied to extract the PSL, such as global or adaptive thresholding [11], $k$-means or fuzzy $c$-means clustering [12], active contours [13], region split and merge [14], morphological operators [15] and edge detection techniques [10]. Thresholding methods perform well when the contrast between the lesion and the skin is good, but fail when the lighting conditions are inhomogeneous. Edge-based methods perform poorly when the image is blurry and when the transition between skin and lesion is smooth. Region-based methods have difficulties when the lesion is textured or inhomogeneous. Moreover, most of these segmentation methods require user interaction and, as mentioned above, many rely on simple photometric features, although shape and texture are clearly important features for this task. When texture is used, parameters are often learned from a small database, and are not easily adapted to a specific image, for example when lighting conditions are inhomogeneous or different to the training set.

We devise an automatic graph-cut segmentation framework with a new data term which takes into account color, texture and shape features learned from the image. For melanoma images in particular, we also use skin chromophores hemoglobin and melanin as additional features extracted from the image. To avoid user-interaction, we derive "seed" pixels from a coarse initial segmentation of the PSL, obtained by clustering and morphological operators. Seed pixels are used by a classifier to derive likelihood probabilities for the final graph-cut delineation of the PSL. 


\section{AUTOMATIC PIGMENTED SKIN LESION DELINEATION}

Graph-cut techniques are among the most powerful methods that extract foreground from background. Initially proposed by [16], graph-cut is an interactive object segmentation approach based on the optimization of a discrete energy function defined on a binary label set $\mathcal{L}=\{0,1\}$ via computing a minimum cut on the image graph. The key task is the proper definition of this energy in order to capture the properties of object regions and those of boundaries between them. This is usually done in the context of maximum a posteriori estimation according to likelihood and prior joint-label distributions so that the minimum of the energy $\hat{l}$ corresponds to the best segmentation among possible labelings $\mathcal{F}$.

Let $\mathcal{G}=\langle\mathcal{V}, \mathcal{E}\rangle$ be the image graph, where each node (pixel) $p$ is assigned a label $l_{p} \in \mathcal{L} . \quad \mathcal{N}_{p}$ is the set of 8connected neighbors of $p$. The labeling solution is given by:

$$
\hat{l}=\underset{l \in \mathcal{F}}{\arg \min }\left(\sum_{p \in \mathcal{V}} D_{p}\left(l_{p}\right)+t \sum_{p \in \mathcal{V}} \sum_{q \in \mathcal{N}_{p}} U_{p, q} \cdot \delta_{l_{p} \neq l_{q}}\right) .
$$

The data term in the fist sum is usually defined by:

$$
D_{p}\left(l_{p}\right)=-\ln \operatorname{Pr}\left(x_{p} \mid l_{p}\right) \approx-\ln h\left(x_{p} ; l_{p}\right)
$$

where $x_{p}$ is the observation $x$ at pixel $p$, often the intensity value. Likelihood probabilities $\operatorname{Pr}\left(x_{p} \mid l_{p}\right)$, which are assumed to be mutually independent, are usually estimated from user-labeled pixels, "seeds", by two normalized histograms $h\left(x_{p} ; l_{p} \in\{0,1\}\right)$, one for the foreground and another for the background. The term $\delta_{l_{p} \neq l_{q}}$ in the second sum is the Potts prior which encourages piecewise-constant labeling. The multiplicative term $U_{p, q}$ is given by:

$$
U_{p, q} \propto \exp \left(-\frac{\left(x_{p}-x_{q}\right)^{2}}{2 \sigma^{2}}\right) \cdot \frac{1}{\operatorname{dist}(p, q)},
$$

where $\sigma$ is a parameter adjusting the sensitivity to intensity difference between neighboring pixels $p$ and $q$, and $\operatorname{dist}(p, q)$ denotes the Euclidean distance between these neighbors.

Graph-cut segmentation has several advantages [17]: globally optimal labeling for $|\mathcal{L}|=2$, practical efficiency, numerical robustness, ability to integrate multiple visual cues and constraints and unrestricted topological properties of regions. Its main drawback is the requirement of user interaction in order define initial seeds, often as hard constrains, meaning that seed pixels cannot change their labels later. For melanoma segmentation, the main task is the definition of the data term (2) in order to take texture and shape features into account, in addition to color. As lighting conditions can change, the model must be adapted to the image to segment, therefore histograms or Gaussian Mixtures cannot be used.

Our method is summarized in Fig. 1. We derive seed pixels automatically from an initial coarse segmentation and define them as soft constraints, so that their labels can change

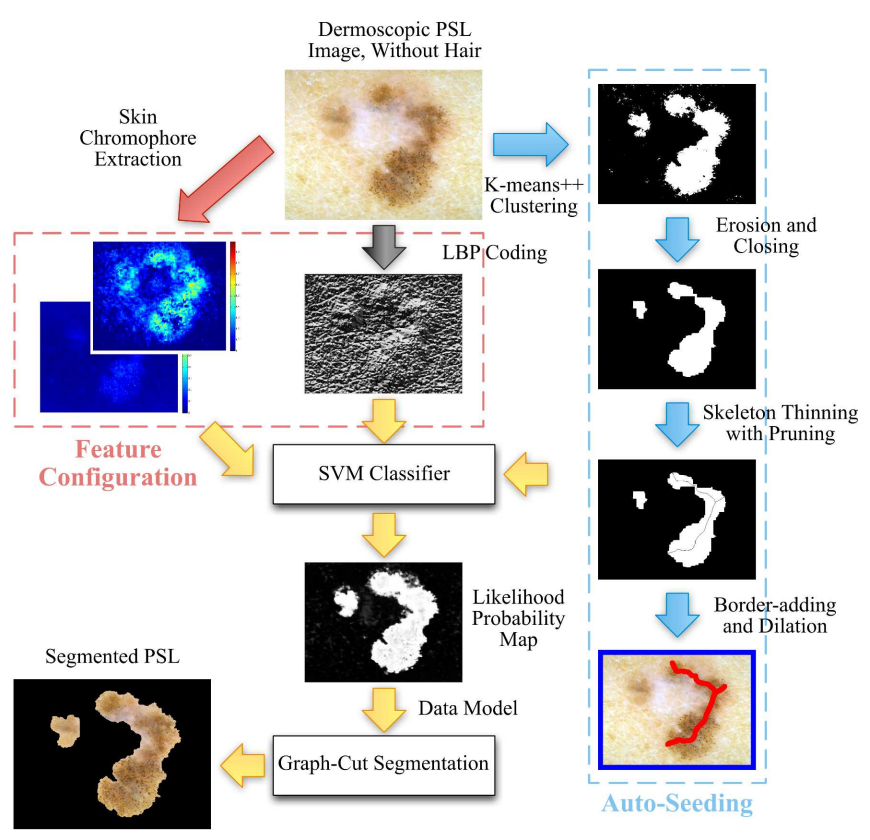

Fig. 1. Flowchart of the proposed automatic pigmented skin lesion segmentation method.

during optimization. Seeds constitute the training set of a supervised classifier, such as the Support Vector Machine (SVM) or the Random Forest (RF), which proceeds on feature vectors of color, shape, texture and chromophores. We define the data term according to the probabilities learned by the classifier.

\subsection{Auto-seeding}

Seed pixels, which are used to train the classifier as well as to initialize graph-cut segmentation, must be representative of the feature distribution. This means that we need a large number of such pixels but must ensure that they belong either to the lesion or the background skin. Pixels near the lesion-skin boundary on either side must be excluded. Hence we define seeds as the central part of an initial coarse segmentation.

First, a preprocessing step suppresses hair using a multiscale method [18]. The eigenvalues $\lambda_{\sigma, 1}, \lambda_{\sigma, 2}$ of the Hessian matrix are computed for each pixel of the image convoluted with a Gaussian kernel at a given scale $\sigma$. The function

$$
\nu(x, y, \sigma)=\exp \left(-\frac{\lambda_{\sigma, 1} / \lambda_{\sigma, 2}}{\beta_{1}}\right)\left(1-\exp \left(-\frac{\sqrt{\lambda_{\sigma, 1}^{2}+\lambda_{\sigma, 2}^{2}}}{\beta_{2}}\right)\right)
$$

is maximum at the center of a hair $x, y$ at a scale $\sigma$ equal to its width. Here $\beta_{1}$ and $\beta_{2}$ are weighting factors. Detection is done with to the following rule:

$$
\operatorname{hair}(x, y)=1, \quad \nu(x, y, \sigma)>\operatorname{mean}_{x, y}\left\{\max _{\sigma}\{\nu(x, y, \sigma)\}\right\}
$$

and hair width at $x, y$ is set to $\sigma$. 
The second step is an unsupervised binary clustering of the image minus detected hairs. We use $k$-means ++ clustering [19] due to its greater robustness to initial values compared to the classical $k$-means algorithm. This produces a coarse segmentation which roughly locates the lesion area along with some small outliers. Sometimes hairs can split some regions in 2 or more parts. Therefore, we apply a morphological closing of a size equal to that of the hair to ensure that the lesions are represented by regions larger than a given area threshold.

Since we want that seed pixels are strictly inside the lesion near its center, we skeletonize the closed coarse segmentation. The skeleton is then pruned by the contour partitioning method in [20], which suppress small branches without displacing the skeleton. A subsequent dilation ensures that the distribution of the seeds includes most of the candidate pixels, from definite inner lesion to possible peripheral lesion pixels.

For background skin seeds, we proceed in a simple manner observing that most skin lesions fall approximately in the center of the dermoscope during acquisition. We define a frame of a given thickness around the image and use pixels inside it as background skin seeds. In the case of large lesions that go beyond the border of the image, we discard frame pixels that overlap with the coarse segmentation of the lesion.

\subsection{New data model}

We replace the classical graph-cut data term derived from seed histograms by a probability delivered by a robust classifier trained on automatically detected seeds. The classifier determines the probability for a feature vector $\mathbf{v}_{p}$ associated with pixel $p$ to be labelled as lesion or background. The vector $\mathbf{v}_{p}$ includes information on color, texture, shape and chromophores rather than scalar intensity only. For each pixel, we start with a feature vector containing its $R, G$ and $B$ values.

\subsubsection{Texture}

Texture information is concatenated to the feature vector of each pixel as a local binary pattern (LBP) [21]. For $N$ (usually 8 ) points on the circumference of a circle of radius $R$ (usually 1) centered at the pixel $p$, the LBP is given by:

$$
\operatorname{LBP}_{N, R}=\sum_{n=0}^{N-1} s\left(L_{n}-L_{p}\right) 2^{n},
$$

where $L_{p}, L_{n}$ denote the luminance values of the pixel $p$ and the point $n$ respectively, and $s(\cdot)$ is a sign function returning 1 if its argument is positive and 0 otherwise.

\subsubsection{Shape}

If the local texture is well represented by the LBP, shape can be represented by a local color template. Therefore, we concatenate the $R, G, B$ values of pixels in a neighborhood of size $M \times M$ around each pixel $p$ to its feature vector $\mathbf{v}_{p}$. Different shapes of lesion border can be captured by this template.

\subsubsection{Chromophores}

Melanocytes are cells that produce a dark chromophore, melanin, which is responsible for the skin color. Melanocytes suffering melanoma exhibit a high concentration of melanin. As the metabolism of these cells increases, the quantity of hemoglobin also increases. We quantify and extract melanin and hemoglobin from $R G B$ images using a Beer-Lambert law based model fitting technique [22]. These two chromophore values are also concatenated to the feature vector.

\subsubsection{Classification and new energy model}

We feed feature vectors constructed for each automatic seed pixel to a classifier, and use its output to derive the likelihood probability defining the data term of graph-cut energy (2). The likelihood gives the probability for a pixel to be lesion or background. Both SVM and RF are robust and efficient classifiers. RF is overfit-free, resistant to outliers and its output is a probability. The decision function of SVM $f(\mathbf{v})$ can be transformed to a probability by fitting a sigmoid model [23]:

$$
P_{A, B}[f(\mathbf{v})] \equiv \frac{1}{1+\exp [A f(\mathbf{v})+B]}
$$

where $A, B$ are constants minimizing the cross entropy error.

The labeling solution (1) thus becomes:

$$
\begin{aligned}
\hat{l} & =\underset{l \in \mathcal{F}}{\arg \min }\left(\sum_{p \in \mathcal{V}}-\ln P_{A, B}[f(\mathbf{v})]\right. \\
& \left.+t \sum_{p \in \mathcal{V}} \sum_{q \in \mathcal{N}_{p}} \frac{1}{\left\|\mathbf{v}_{p}-\mathbf{v}_{q}\right\|^{2}+1} \cdot \frac{\delta_{l_{p} \neq l_{q}}}{\operatorname{dist}(p, q)}\right) .
\end{aligned}
$$

where $\|\cdot\|^{2}$ denotes the $L^{2}$ norm.

We note that we use seed pixels as "soft" labeling constraints as $D_{p}\left(l_{p}\right)=-\ln P_{A, B}[f(\mathbf{v})]$. In classical graph-cut segmentation [16], seeds are used as "hard" constraints for pixels that cannot change their initial label, since $D_{p}\left(l_{p}\right)$ is defined by a large constant when $p$ is a seed.

\section{EXPERIMENTAL RESULTS}

\subsection{Natural images}

We first compare our method on natural images with three graph-cut segmentation methods: the classical method (BJ) [16], the Lazy Snapping algorithm (LS) [24] and the Grab-Cut algorithm (GBC) [25]. Both qualitative (Fig. 2) and quantitative (Table 1) evaluations confirm the importance of shape and texture features. Using these features, our method is able to differentiate an object from the background even if their intensity distributions are very similar, such as the building and the cross in Fig. 2. In quantitative evaluation, we use the same seeds for every method, except for GBC. We can 


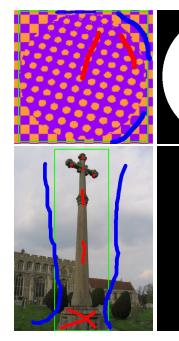

(a)

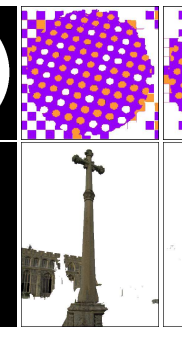

(c)

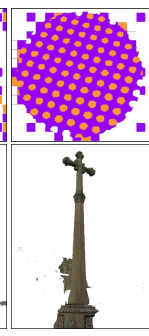

(d)

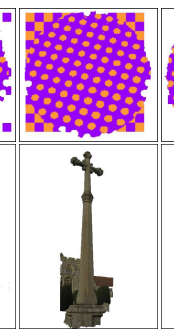

(e)

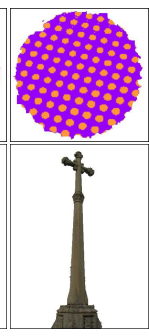

(f)

Fig. 2. Qualitative comparison on a synthetic texture (top) and a natural image (bottom). (a) Initial image with seeds, (b) ground-truth, (c) BJ, (d) LS, (e) GBC, (f) our approach.

\begin{tabular}{|c|c|c|c|c|}
\hline & BJ & LS & GBC & Our \\
\hline Texture & 27.46 & 11.65 & 19.46 & $\mathbf{3 . 8 1}$ \\
\hline Leopard & 37.22 & 55.95 & 47.16 & $\mathbf{1 1 . 8 4}$ \\
\hline Grave & 20.63 & 10.89 & $\mathbf{5 . 1 5}$ & 7.61 \\
\hline Cross & 75.81 & 18.43 & 57.82 & $\mathbf{3 . 9 9}$ \\
\hline Swimmer & 16.25 & $\mathbf{8 . 0 2}$ & 165.20 & 13.10 \\
\hline Plane & 15.59 & 19.00 & 38.10 & $\mathbf{6 . 7 8}$ \\
\hline Japanese & 6.33 & 5.29 & 3.58 & $\mathbf{1 . 5 2}$ \\
\hline Sheep & 55.23 & 70.97 & 51.43 & $\mathbf{2 0 . 6 6}$ \\
\hline Birds & 19.05 & 18.64 & 25.50 & $\mathbf{1 0 . 8 2}$ \\
\hline Boat & 18.60 & 17.17 & 10.58 & $\mathbf{9 . 6 4}$ \\
\hline
\end{tabular}

Table 1. Segmentation error on natural color images from the Berkeley [26] and the MSRC [27] segmentation datasets.

see that the error rate, reported as the percentage of wrongly segmented pixels, is lower for our approach.

\subsection{Melanoma images}

We next evaluate our method on real dermoscopic images. Fig. 3 illustrates automatically defined seeds on several images from the EDRA dataset [28]. Lesion seeds are in red, and background seeds in blue. Results of qualitative and quantitative comparison with other graph-cut methods on this dataset are given in Fig. 4 and Table 2 respectively. Quantitative measures used are the Dice coefficient, the recall and the precision values all reported on 100 dermoscopic images. We can see that our method outperforms other graph-cut methods in terms of Dice and is comparable to others on other measures.

\section{CONCLUSIONS}

We presented a new data model for graph-cut image segmentation, defined according to probabilities learned by a classification process, which takes into account not only color but also texture and shape information. For melanoma segmentation, we also introduce skin chromophore features and automatically derive "seed" pixels used to train the classifier from
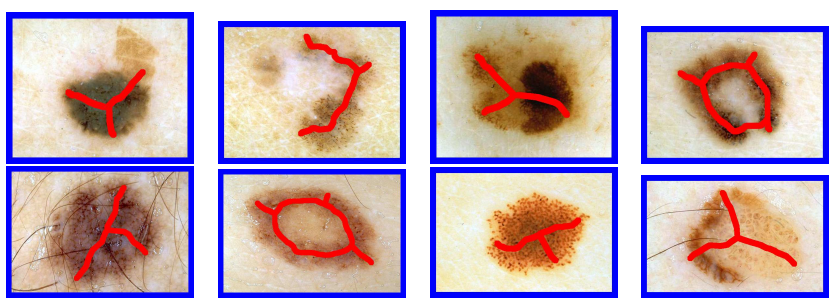

Fig. 3. Automatic seeds on melanoma (top) and on dysplastic nevi (bottom) images.
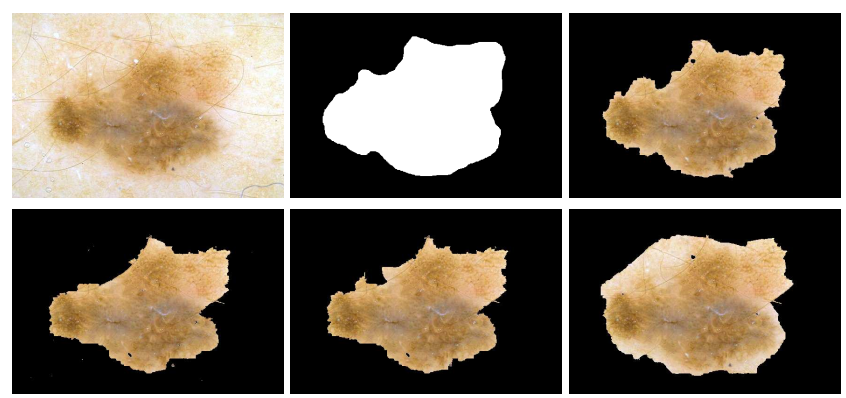

Fig. 4. Melanoma delineation. Left to right, top to bottom: dermoscopic image, ground truth, our method, LS, BJ, GBC.

\begin{tabular}{|c|c|c|c|c|c|c|}
\hline & \multicolumn{2}{|c|}{ Dice } & \multicolumn{2}{c|}{ Precision } & \multicolumn{2}{c|}{ Recall } \\
\cline { 2 - 7 } & mean & s.d. & mean & s.d. & mean & s.d. \\
\hline \hline Our & $\mathbf{9 3 . 8 5}$ & $\mathbf{3 . 0 8}$ & 98.36 & 1.68 & 88.27 & 7.56 \\
LS & 88.73 & 4.68 & 98.76 & 1.53 & 80.89 & 7.67 \\
BJ & 88.70 & 5.00 & $\mathbf{9 9 . 0 7}$ & $\mathbf{0 . 8 1}$ & 80.66 & 8.14 \\
GBC & 87.90 & 9.90 & 84.37 & 16.45 & $\mathbf{9 5 . 5 3}$ & $\mathbf{4 . 0 8}$ \\
\hline
\end{tabular}

Table 2. Quantitative evaluation of melanoma delineation on 100 dermoscopic images from the EDRA dataset.

a coarse initial segmentation. On natural images, our method successfully segments objects with similar color distributions but different textures. Its application to melanoma delineation in particular gives promising results.

We are currently investigating the extension of our method to multispectral dermoscopic images which, in addition to $R G B$, include 4 additional wavelengths of which 2 are infrared. The focus is therefore on adapting extracted texture, shape and chromophore features to the new space.

\section{REFERENCES}

[1] A. Jemal, T. Murray, E. Ward, A. Samuels, R. Tiwari, A. Ghafoor, E. Feuer, and M. Thun, "Cancer statistics, 2005," CA: A Cancer Journal for Clinicians, vol. 55, no. 1, pp. 10-30, 2005.

[2] D. Rigel, R. Friedman, A. Kopf, and D. Polsky, "Abcdean evolving concept in the early detection of melanoma," Archives of dermatology, vol. 141, no. 8, pp. 1032-1034, 2005. 
[3] G. Argenziano, G. Fabbrocini, P. Carli, V. De Giorgi, E. Sammarco, and M. Delfino, "Epiluminescence microscopy for the diagnosis of doubtful melanocytic skin lesions: comparison of the abcd rule of dermatoscopy and a new 7-point checklist based on pattern analysis," Archives of Dermatology, vol. 134, no. 12 , pp. 1563-1570, 1998.

[4] M. Binder, H. Kittler, S. Dreiseitl, H. Ganster, K. Wolff, and H. Pehamberger, "Computer-aided epiluminescence microscopy of pigmented skin lesions: the value of clinical data for the classification process," Melanoma research, vol. 10, no. 6, pp. 556-561, 2000.

[5] E. Celebi, G. Schaefer, H. Iyatomi, and W. Stoecker, "Lesion border detection in dermoscopy images," Computerized medical imaging and graphics, vol. 33, no. 2, pp. 148, 2009.

[6] M. Faal, M. H. Miran Baygi, and E. Kabir, "Improving the diagnostic accuracy of dysplastic and melanoma lesions using the decision template combination method," Skin Research and Technology, vol. 19, no. 1, pp. e113-e122, 2013.

[7] H. Ganster, A. Pinz, R. Rohrer, E. Wildling, M. Binder, and H. Kittler, "Automated melanoma recognition," IEEE Transactions on Medical Imaging, vol. 20, no. 3, pp. 233-239, 2001.

[8] E. Celebi, Y. Alp Aslandogan, W. Stoecker, H. Iyatomi, H. Oka, and X. Chen, "Unsupervised border detection in dermoscopy images," Skin Research and Technology, vol. 13, no. 4, pp. 454-462, 2007.

[9] P. Schmid, "Segmentation of digitized dermatoscopic images by two-dimensional color clustering," IEEE Transactions on Medical Imaging, vol. 18, no. 2, pp. 164-171, 1999.

[10] H. Zhou, M. Chen, R. Gass, J. M. Rehg, L. Ferris, J. Ho, and L. Drogowski, "Feature-preserving artifact removal from dermoscopy images," in SPIE Medical Imaging, 2008, vol. 6914, pp. 1-9.

[11] T. Mendonca, A. Marcal, A. Vieira, J. C. Nascimento, M. Silveira, J. S. Marques, and J. Rozeira, "Comparison of segmentation methods for automatic diagnosis of dermoscopy images," in IEEE Int. Conf. of the Engineering in Medicine and Biology Society (EMBS), 2007, pp. 6572-6575.

[12] M. E. Yuksel and M. Borlu, "Accurate segmentation of dermoscopic images by image thresholding based on type-2 fuzzy logic," IEEE Transactions on Fuzzy Systems, vol. 17, no. 4, pp. 976-982, 2009.

[13] B. Erkol, R. H. Moss, S. R. Joe, W. V. Stoecker, and E. Hvatum, "Automatic lesion boundary detection in dermoscopy images using gradient vector flow snakes," Skin Research and Technology, vol. 11, no. 1, pp. 17-26, 2005.

[14] E. Celebi, H. Kingravi, H. Iyatomi, Y. Alp Aslandogan, W. Stoecker, R. Moss, J. Malters, J. Grichnik, A. Marghoob, and H. Rabinovitz, "Border detection in dermoscopy images using statistical region merging," Skin Research and Technology, vol. 14, no. 3, pp. 347-353, 2008.
[15] A. Beuren, R. Janasieivicz, P. Gomes, G. Neusa, and J. Facon, "Skin melanoma segmentation by morphological approach," in Proc. of the Int. Conf. on Advances in Computing, Communications and Informatics (ICACCI), 2012, pp. 972-978.

[16] Y. Boykov and G. Funka-Lea, "Graph cuts and efficient nd image segmentation," International Journal of Computer Vision, vol. 70, no. 2, pp. 109-131, 2006.

[17] R. Kéchichian, S. Valette, M. Desvignes, and R. Prost, "Shortest-path constraints for 3d multiobject semiautomatic segmentation via clustering and graph cut," IEEE Transactions on Image Processing, vol. 22, no. 11, 2013.

[18] O. Tankyevych, H. Talbot, and P. Dokladal, "Curvilinear morpho-hessian filter," in IEEE Int. Symp. on Biomedical Imaging (ISBI), 2008, pp. 1011-1014.

[19] D. Arthur and S. Vassilvitskii, "k-means++: The advantages of careful seeding," in Proc. of the ACM-SIAM symposium on Discrete Algorithms, 2007, pp. 1027-1035.

[20] X. Bai, L. J. Latecki, and W.-Y. Liu, "Skeleton pruning by contour partitioning with discrete curve evolution," IEEE Transactions on Pattern Analysis and Machine Intelligence, vol. 29, no. 3, pp. 449-462, 2007.

[21] T. Ojala, M. Pietikainen, and T. Maenpaa, "Multiresolution gray-scale and rotation invariant texture classification with local binary patterns," IEEE Transactions on Pattern Analysis and Machine Intelligence, vol. 24, no. 7, pp. 971-987, 2002.

[22] H. Gong and M. Desvignes, "Quantification of pigmentation in human skin images," in Proc. of IEEE Int. Conf on Image Processing (ICIP), 2012, pp. 2853-2856.

[23] J. Platt, "Probabilistic outputs for support vector machines and comparisons to regularized likelihood methods," Advances in Large Margin Classifiers, vol. 10, no. 3, pp. 61-74, 1999.

[24] Y. Li, J. Sun, C.-K. Tang, and H.-Y. Shum, "Lazy snapping," ACM Transactions on Graphics, vol. 23, no. 3, pp. 303-308, 2004.

[25] C. Rother, V. Kolmogorov, and A. Blake, "Grabcut: Interactive foreground extraction using iterated graph cuts," ACM Transactions on Graphics, vol. 23, no. 3, pp. 309-314, 2004.

[26] Martin D., Fowlkes C., Tal D., and Malik J., "A database of human segmented natural images and its application to evaluating segmentation algorithms and measuring ecological statistics," in Proc. of the Int. Conf. on Computer Vision (ICCV), 2001, vol. 2, pp. 416-423.

[27] J. Shotton, J. Winn, C. Rother, and A. Criminisi, "TextonBoost for image understanding: Multi-class object recognition and segmentation by jointly modeling texture, layout, and context," International Journal of Computer Vision, vol. 81, no. 1, pp. 2-23, 2009.

[28] G. Argenziano, H. P. Soyer, V. De Giorgi, D. Piccolo, P. Carli, and M. Delfino, "Dermoscopy: a tutorial," EDRA, Medical Publishing \& New Media, 2002. 